\title{
Some Results on the Qualitative Theory of Matrix ${ }^{\dagger}$
}

\section{Yoshiteru IsHIDA*, Norihiko ADACHI* and Hidekatsu TOKUMARU*}

\begin{abstract}
In this paper, we discuss the sign stability and the potential stability for the linear time invarient system described by

$d x / d t=A x, \quad x \in R^{n}, \quad A \in R^{n \times n}$.

The necessary and sufficient conditions for sign stability are obtained. The sufficient conditions for potential stability are also discussed with the concept of cycle decomposition. The results obtained are more general than those so far known. The concept of inertia preservation, which is the generalization of sign stability, is introduced to classify matrices from the structural point of view. Some system structures to preserve inertia are shown.
\end{abstract}

\section{Introduction}

We are concerned with the dynamics of a linear system described by the equation

$$
d x / d t=A x, \quad x \in R^{n}, \quad A \in R^{n \times n} .
$$

A property of the system is called structural when it depends on the sign pattern $(+,-, 0)$ of each element of matrix. On the other hand if the properties depends on the values of the elements, they are called parametric properties. It is important in system analysis or synthesis of system to distinguish structural properties from parametric ones in the following three points.

(i) As is often the case in the fields of econometrics, ecology or chemistry, the information is insufficient to identify the value of each element of matrix $A$. For example, suppose there is the community of $n$-species in equilibrium. Then the

†第 22 回自動制御連合講演会で発表（昭 $54 \cdot 10$ )

* 京都大学工学部 京都市左京区吉田本町

* Faculty of Engineering, Kyoto University, Kyoto (Received March 11, 1980)

(Revised June 16, 1980) first order effects among these species will be described by the equation

$$
d x_{i} / d t=\sum_{j=1}^{n} a_{i j} x_{j}(i=1, \cdots, n)
$$

where $x_{i}$ represents the growth of population of the $i$ th species and $\left\{a_{i j}\right\}$ is the interaction matrix. In this case value can not be measured exactly. But we can know some structural information such that if $i$ th species is self-regulating then $a_{i}<<0$ or if there is the predator-prey relation between the $i$ th and the $j$ th species then $a_{i j} a_{j i}<0$. In these case, the stability, the observability and the singularity of matrix can be known to some extent only with its graph.

(ii) Even in the case that the value of the element of the matrix can be known, the structural properties such as sign stability or potential stability can reduce the numerical efforts to calculates its eigenvalue.

(iii) Furthermore, it is useful to know the element of the matrix which contributes to the stability or to the instability of the system. For example, the matrix with the following sign pattern will be stable or unstable dependently on the absolute values of the nonzero elements,

$$
\left\{a_{i j}\right\}=\left[\begin{array}{cc}
- & - \\
+ & - \\
0 & +
\end{array}\right]
$$

In this case, by replacing $a_{13}$ and $a_{22}$ by 0 , the system with this sign pattern is always stable. It is to be noted that setting a subsystem more stable may contribute to the instability of the whole system. Indeed, in this example, the larger absolute value of $a_{11}$ make the whole system unstable.

The main theme of this paper is to reseach on the stability from the structural viewpoint using 

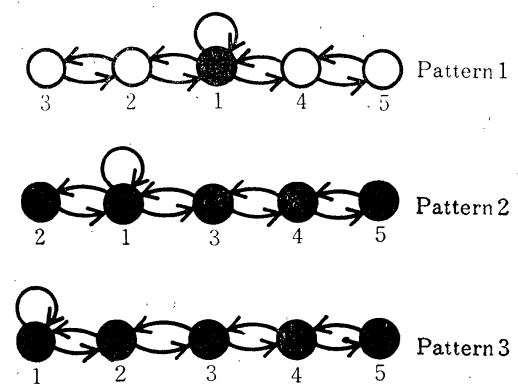

Fig. 1 The graph of matrices $A \in R^{5 \times 5}$ which satisfy the condition 1),2) and 3 )

The color test has already been executed to them.

its graphical representation.

\section{【Definition 0】}

The graph $G(V, U)$ of matrix $A \in R^{n \times n}$ is the pair of the two sets $(V, U)$ where $V$ is the set of $n$ vertices which correspond to $n$-elements of $x \in R^{n}$, and $U$ is the set of arcs. The arc $e_{i j} \in U$ is directed from vertex $j$ to vertex $i$ if and only if $a_{i j} \neq 0$.

Matrix $A$ is said to be sign stable if every matrix which has the sign pattern identical with that of $A$ is stable (that is, the real part of every eigenvalue of the matrix is negative). The following conditions for matrix $A \in R^{n \times n}$ to be stable are proposed by J. Quirk ${ }^{5}$.

1) $a_{k k} \leqq 0$ for every $k, a_{i i}<0$ for some $i$.

2) $a_{i j} a_{j i} \leqq 0$ for every $i \neq j$.

3) Every cycle in the graph of matrix $A \in R^{n \times n}$ of length greater than two is zero.

4) $\operatorname{det} A \neq 0$

A counter example showed that these conditions are insufficient. The example (Fig. 1, pattern 1) was presented by C. Jefferies ${ }^{1)}$. And the necessary and sufficient conditions for sign stability were given by the same author ${ }^{3)}$.

In section 2, a concept of sign observability for linear system is introduced. And this concept gives explicitly analytical expressions of the necessary and sufficient conditions for the sign stability. Some examples are given to show how our conditions relate to those of him. In section 3 , the potential stability of a matrix, which is also one of the important concepts in the qualitative theory of linear system is discussed. A matrix is said to be potentially stable if there exists a stable matrix whose sign pattern is identical with that of $A$. Sufficient conditions for potential stability are proposed. The conditions are more general than the following so far known condition ${ }^{6}$.

《Theorem (Quirk)》

A sufficient condition for matrix $A \in R^{n \times n}$ to be potentially stable is that all the diagonal elements of $A$ are negative.

In section 4, inertia preserving sign patterns are discussed. Qualitative matrix of $A$ is the set of all matrices whose sign patterns are identical with that of $A$. And the matrix $A$ is said to preserve its inertia when every element of the qualitative matrix of $A$ has the same sign pattern of the real parts of all the eigenvalues.

The sign stable sign patterns are induced in the inertia preserving sign patterns. In section 5 , with the concept of preceding sections, matrices $A \in R^{n \times n}$ are classified from the qualitative point of view. And this classification gives the clear position of sign stable matrix and potentially stable matrix in the set of matrices $A \in R^{n \times n}$. The complete classification of matrices $A \in R^{2 \times 2}$ is shown as an example.

\section{Sign stability}

\section{【Definition 1】}

Matrix $A \in R^{n \times n}$ is said to be decomposable if $A$. can be permuted into the form:

$$
\left[\begin{array}{cc}
A_{11} & A_{12} \\
0 & A_{22}
\end{array}\right]
$$

where $A_{11}$ and $A_{22}$ are square blocks, otherwise $A$ is said to be indecomposable.

It suffices to consider only the indecomposable case, for when a matrix is decomposable, it can be decomposed into indecomposable square blocks.

And the eigenvalues of the matrix are the union of the eigenvalues of its indecomposable square blocks. Hereafter, matrix $A \in R^{n \times n}$ under consideration is assumed to be indecomposable.

\section{【Definition 2】}

Matrix $A \in R^{n \times n}$ is said to be sign similar to $B$ $\in R^{n \times n}$ if $\operatorname{sgn} A=\operatorname{sgn} B$, where $\operatorname{sgn} A=\left(a_{j k}{ }^{*}\right), a_{j k} *$ $=\operatorname{sgn} a_{j k}$

where $\operatorname{sgn} a_{j k}=\left\{\begin{array}{r}1 \text { if } a_{j k}>0 \\ -1 \text { if } a_{j k}<0 \\ 0 \text { if } a_{j k}=0\end{array}\right.$ 
And let $Q_{A}=\{B \mid \operatorname{sgn} B=\operatorname{sgn} A\}$ be called the qualitative matrices of $A$.

\section{【Definition 3】}

Matrix $A$ is said to be stable if all the real parts of its eigenvalues are negative. Matrix $A \in R^{n \times n}$ is said to be sign stable if $B \in Q_{A}$ implies that $B$ is stable.

【Definition 4】 (cycle and loop of a matrix)

Matrix $A$ is said to have $k$-cycle (loop) if the graph of $A$ has $k$-circuit $e_{i_{1} i_{2}}, e_{i_{2} i_{3}}, \cdots, e_{i_{k} i_{1}}\left(e_{i_{1} i_{1}}\right.$, respectively).

\section{【Definition 5】}

Let $(A, C), A \in R^{n \times n}, C \in R^{m \times n}$ be the pair of two matrices. $(A, C)$ is called sign observable if $(\hat{A}, \hat{C})$ is observable for every $\hat{A} \in Q_{A}$ and $\hat{C} \in Q c$.

With the concept of sign observability, the necessary and sufficient conditions for a matrix to be sign stable are obtained in the following theorem.

\section{《Theorem 1》}

Matrix $A \in R^{n \times n}$ is sign stable if and only if 1 ), 2), 3) and 4) hold.

1) $a_{k k} \leqq 0$ for every $k, a_{i i}<0$ for some $i$.

2) $a_{i j} a_{j i} \leqq 0$ for every $i \neq j$.

3) Every cycle in the graph of matrix $A \in R^{n \times n}$ of length greater than two is zero.

$4)^{\prime}\left(A_{22}, A_{12}\right)$ is sign observable when $A$ is permuted into the form: $\left[\begin{array}{ll}A_{11} & A_{12} \\ A_{21} & A_{22}\end{array}\right]$

where all the diagonal elements of $A_{11}$ are negative and those of $A_{22}$ are zeros. proof (Appendix 1)

The equivalent conditions for sign stability have already been proposed by C. Jefferies ${ }^{1)}$, using the graphic method named color test.

The conditions are as follows:

1), 2),3) and the following 4)" are satisfied.

4)" The graph of $A$ fails in the color test, and $\operatorname{det} A \neq 0$.

The graph of $A$ is said to pass the color test when every vertex can be colored to satisfy the following conditions :

(a) Each vertex which has loop is black.

(b) There is at least one white vertex in the graph.

(c) Each white vertex is connected to at least one other white vertex.

(d) Each black vertex connected to one white vertex is connected to at least one other white vertex.

In this color test, black vertex corresponds to non-oscillating elements of $x \in R^{n}$ and white vertex corresponds to oscillating element. Thus, if the graph of a matrix passes the color test, there exists some $\hat{A} \in Q_{A}$ which has pure imaginaries as its eigenvalues. As for the condition $\operatorname{det} A \neq 0$, it can be checked easily by examining the matching of the graph of the matrix ${ }^{2), 3}$.

Let us show some examples which make clear the relation between condition 4$)^{\prime}$ and 4$)^{\prime \prime}$.

\section{[Example 1]}

In Fig. 1 , the graphs of matrices $A \in R^{5 \times 5}$ which satisfy the condition 1),2) and 3) are shown. The color test has already been executed to them. The matrices are permuted into the form of the condition 4$)^{\prime}$. In the case of pattern 1 , the matrix $\hat{A}$ and its observability matrix $K=\left[A_{12}, A_{12} A_{22}\right.$, $\left.A_{12} A_{22}{ }^{2}, A_{12} A_{22^{3}}\right]^{t}$ are as follows:

$$
\begin{aligned}
& \hat{A}=\left[\begin{array}{ccccc}
-a_{11} & a_{12} & 0 & a_{14} & 0 \\
-a_{21} & 0 & -a_{23} & 0 & 0 \\
0 & a_{32} & 0 & 0 & 0 \\
-a_{41} & 0 & 0 & 0 & -a_{45} \\
0 & 0 & 0 & a_{54} & 0
\end{array}\right] \\
& K=\left[\begin{array}{cccc}
a_{12} & 0 & a_{14} & 0 \\
0 & -\alpha & 0 & -\beta \\
-\alpha a_{32} & 0 & -\beta a_{54} & 0 \\
0 & \alpha \gamma & 0 & \beta \delta
\end{array}\right]
\end{aligned}
$$

where $a_{i j}>0(i=1, \cdots, 5, j=1, \cdots, 5)$

$$
\alpha=a_{12} a_{23}, \beta=a_{14} a_{45}, \gamma=a_{32} a_{23}, \delta=a_{45} a_{54}
$$

The matrix $A$ is not sign observable, for $\operatorname{det} K$ $=0$ when $a_{23} a_{32}=a_{45} a_{54}$. Therefore, the graph of $A$ passes the color test though det $\hat{A} \neq 0$ for every $\hat{A} \in Q_{A}$. The matrix $A$ and its observability matrix have the following form, in the case of pattern 2 .

$$
\begin{aligned}
& \hat{A}=\left[\begin{array}{ccccc}
-a_{11} & a_{12} & a_{13} & 0 & 0 \\
-a_{21} & 0 & 0 & 0 & 0 \\
-a_{31} & 0 & 0 & a_{34} & 0 \\
0 & 0 & -a_{43} & 0 & a_{45} \\
0 & 0 & 0 & -a_{54} & 0
\end{array}\right] \\
& K=\left[\begin{array}{cccc}
a_{12} & a_{13} & 0 & 0 \\
0 & 0 & \varepsilon & 0 \\
0 & -\varepsilon a_{43} & 0 & \varepsilon a_{45} \\
0 & 0 & -\varepsilon(\phi+\delta) & 0
\end{array}\right]
\end{aligned}
$$

where $a_{i j}>0(i=1, \cdots 5, j=1, \cdots, 5)$

$$
\varepsilon=a_{13} a_{34}, \quad \phi=a_{34} a_{45}
$$


52 Trans. of the Society of Instrument and Control Engineers Vol. 17, No. 1 February 1981

In this case, though the graph fails in the color test, $\hat{A}$ is not sign observable, for $\operatorname{det} \hat{A}=0$ for every $\hat{A}, \hat{A} \in Q_{A}$. The sign non observability of this case is clearly shown in the form of matrix $K$, too. In the case of pattern 3 , the matrix $A$ and $K$ are as follows.

$$
\begin{aligned}
& A=\left[\begin{array}{ccccc}
-a_{11} & a_{12} & 0 & 0 & 0 \\
-a_{21} & 0 & a_{23} & 0 & 0 \\
0 & -a_{32} & 0 & a_{34} & 0 \\
0 & 0 & -a_{43} & 0 & a_{45} \\
0 & 0 & 0 & -a_{54} & 0
\end{array}\right] \\
& K=\left[\begin{array}{cccc}
a_{12} & 0 & 0 & 0 \\
0 & \alpha & 0 & 0 \\
-\alpha a_{32} & 0 & \alpha a_{34} & 0 \\
0 & -\alpha(\gamma+\eta) & 0 & \alpha \phi
\end{array}\right]
\end{aligned}
$$

where $a_{i j}>0(i=1, \cdots, 5, j=5) \eta=a_{34} a_{43}$

This is only the case the matrix is sign observable because the matrix $K$ has clearly full rank.

\section{Potential stability}

The possibility for a matrix to be sign stable is very small and if it fails to satisfy the conditions of Theorem 1, the only way to know whether the system is stable with its graph is to check potential stability. Therefore the concept of potential stability is of great importance. Potential instability of $A$ implies that $A$ is unstable for every $\hat{A} \in Q_{A}$, thus the conditions for potential stability gives the way to see whether the system is unstable or not without calculating its eigenvalues.

\section{【Definition 6】}

Matrix $A \in R^{n \times n}$ is said to be potentially stable if there exists $\hat{A} \in Q_{A}$ such that $\hat{A}$ is stable.

\section{【Definition 7】}

The sign of $k$-cycle $a_{i_{1} i_{2}} a_{i_{2} i_{3}}, \cdots, a_{i_{k} i_{1}}$ means $\operatorname{sgn}\left(a_{i_{1} i_{2}} a_{i_{2} i_{3}}, \cdots, a_{i_{k} i_{1}}\right)$.

【Definition 8】 (cycle decomposition)

Matrix $A \in R^{n \times n}$ can be decomposed uniquely as follows : $A=C_{-}+R$ where $C_{-} \in R^{n \times n}$ is the matrix of all the negative cycles of $A$ and $R \in R^{n \times n}$ is that of remaining elements. And furthermore $A, C$ - and $R$ have the graph $(V, U),\left(V, U_{1}\right)$ and $\left(V, U_{2}\right)$ respectively such that $U=U_{1} \cup U_{2}, U_{1} \cap U_{2}=\phi$. The decomposition is called cycle decomposition.

The main result of this section is the following theorem which gives sufficient conditions for a matrix to be potentially stable.

\section{《Theorem 2》}

Let $C_{-}=C_{-}[1,2]+P$, where $C_{-}[1,2]$ is the matrix of all 1 and 2 cycle of $C_{-}, P$ is that of remaining elements of $C_{-}$, and the graph of $C_{-}[1,2]$ and that of $P$ do not share the same edge.

Matrix $A=C_{-}+R$ is potentially stable if $C_{-}[1,2]$ is sign stable.

This theorem directly follows from the fact that the eigenvalues of $A$ moves continuously on the complex plane if the coefficients of the characteristic equation of $A$ changes continuously.

As for the necessary condition for potential stability, the following theorem (which has already proposed in 4)) are obtained using the cycle decomposition.

【Definition 9】( $G[n]$-cycle)

$G[n]$-cycle is a set of $k$-cycles $(k \leqq n)$ such that the sum of the length of this set is $n$ and any two cycles of this set do not share the same vertex.

\section{《Theorem 3》}

If matrix $A \in R^{n \times n}$ is potentially stable then $C_{\text {- }}$ has a nonzero $G[k]$-cycle for every $k$, where $C_{-}$is the matrix of negative cycles in the cycle decomposition : $A=C_{-}+P$

\section{[Example 2]}

This example (Fig. 2) satisfies the condition of Theorem 3 , that is, this system has $G[k]$-cycle $(k=$ $1, \cdots, 4) . \quad G[1]-$ cycle $=\left\{a_{11}\right\}, \quad G[2]-$ cycle $=\left\{a_{23} a_{32}\right\}$, $G[3]$-cycle $=\left\{a_{11}, a_{23} a_{32}\right\}, G[4]-$ cycle $=\left\{a_{12} a_{23} a_{34} a_{41}\right\}$.

But this system is unstable because this condition ensures only that each coefficient of the characteristic equation of this system is positive, in fact, the Hurwizian matrix is

$$
H_{4}=\left[\begin{array}{cccc}
-a_{11} & \alpha a_{11} & 0 & 0 \\
1 & -\alpha & -\beta & 0 \\
0 & -a_{11} & \alpha a_{11} & 0 \\
0 & 1 & -\alpha & -\beta
\end{array}\right]
$$

and det $H_{4}=-\left(\beta a_{11}\right)^{2}$, where $\alpha=a_{23} a_{32}$ $\beta=a_{12} a_{23} a_{34} a_{41}$.

\section{[Example 3]}

This example (Fig. 3) shows the lack of necessity of the condition of Theorem 2. This system does not satisfy the condition, for the matrix $C$ - is decomposed into $C_{-}[1,2]$ and $P$, and $C_{-}[1,2]$ is 


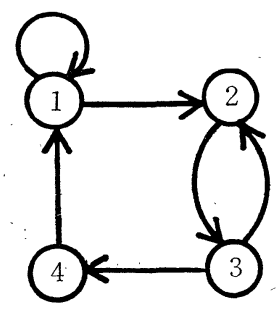

Fig. 2 The example which satisfies the condition of Theorem 3

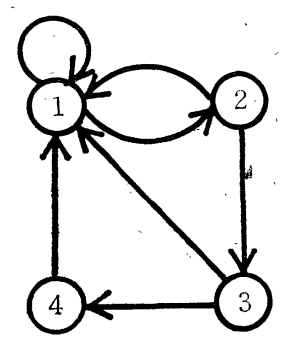

Fig. 3 The example which shows the lack of necessity of the condition of Theorem 2

not sign stable.

$$
A=\left[\begin{array}{cccc}
-a_{11} & -a_{12} & -a_{13} & -a_{14} \\
a_{21} & 0 & 0 & 0 \\
0 & { }^{-1} a_{32} & 0 & 0 \\
0 & 0 & a_{43} & 0
\end{array}\right]
$$

where $a_{i j}>0(i=1, \cdots, 4, j=1, \cdots, 4)$

$A=C_{-}$

$$
C-[1,2]=\left[\begin{array}{cccc}
-a_{11} & -a_{12} & 0 & 0 \\
a_{21} & 0 & 0 & 0 \\
0 & 0 & 0 & 0 \\
0 & 0 & 0 & 0
\end{array}\right]
$$

But this system is clearly potentially stable because the characteristic equation:

$$
\lambda^{4}-a_{11} \lambda^{3}-a_{12} a_{21} \lambda^{2}-a_{12} a_{23} a_{31} \lambda-a_{12} a_{23} a_{34} a_{41}=0
$$

can have any possible 4-roots of negative real parts if $a_{i j}$ changes. It is also clear, that this system satisfies the condition of Theorem 3 .

\section{Inertia preserving sign pattern}

\section{(Definition 10】 ${ }^{4), 7)}$}

The inertia of matrix $A$ is defined as a triple of nonnegative integers $\operatorname{In}(A)=(p, n, i)$ where $p \equiv p(A)$ is the number of the eigenvalues of $A$ with positive real parts, $n \equiv n(A)$ with negative real parts, $i \equiv i(A)$ with zero real parts.

$$
\left(A \in R^{m \times m}, \quad p+n+i=m\right) \text {. }
$$

\section{【Definition 11】}

Let matrix $A \in R^{m \times m}$ be called inertia preserving if $\operatorname{In}(\hat{A})=\operatorname{In}(A)$ for every $\hat{A} \in Q_{A}$. And let the class of matrices $\left\{A \mid \operatorname{In}(\hat{A})=(p, n, i)\right.$ for every $\left.\hat{A} \in Q_{A}\right\}$ be denoted by $I(p, n, i)$.

Preservation of inertia is a generalization of the sign stability because matrix $A$ is sign stable implies $A \in I(0, m, 0)$ and vice versa. In this section sufficient conditions for matrix $A \in R^{n \times n}$ to be inertia preserving are obtained from the OstrowskiSchneider's theorem ${ }^{4)}$.

《Theorem 4》》

Matrix $A \in R^{n \times n}$ belong, to $I(q, p, 0)$ if there exists the matrix $E_{p q}$ such that $E_{p q} A$ is sign stable with $a_{i i}<0 \quad(i=1, \cdots, n)$, where $E_{p q} \in R^{n \times n}$ is the matrix $e_{i j}$ such that $e_{i j}=0 \quad(i \neq j), e_{i_{k} i_{k}}=1 \quad(k=1$, $\cdots, p),=-1(k=p+1, \cdots, p+q)$. proof (Appendix 2)

The other two sign patterns for a matrix to be inertia preserving are obtained in the following two theorems.

\section{《Theorem 5》}

If the graph of matrix $A \in R^{n \times n}$ is a $n$-cycle then $A$ is inertia preserving (Fig. 4).

This theorem is trivial, for the characteristic equation of $A$ is of the form :

$$
\lambda^{n}=a_{i_{1} i_{2}} a_{i_{2} i_{3}} \cdots a_{i n i_{1}}
$$

《Theorem 6》》

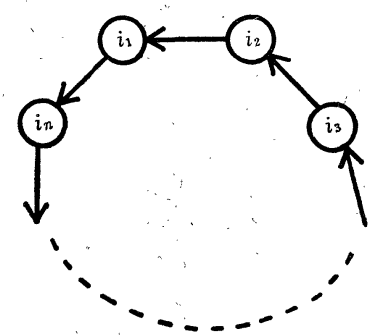

Fig. 4 The graph of $n$-cycle

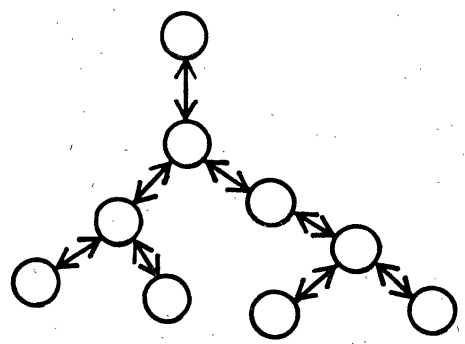

Fig. 5 The graph without any loop and cycle of length greater than two and sign of every two cycle is negative 
If the graph of matrix has no loop and no cycle of length greater than two, and if every sign of 2-cycle is negative then $A$ belongs to $I(0,0, n)$ (Fig. 5). proof (Appendix 3)

\section{The classification of a matrix}

Let the class of matrices $\{A \mid \operatorname{In}(\hat{A})=(p, n, i)$ for some $\left.\hat{A} \in Q_{A}\right\}$ be denoted by $P(p, n, i)$. From the same arguments as for the Theorem 2, the next theorem follows immediately.

\section{《Theorem 7》}

Matrix $A \in R^{n \times n}$ belongs to $P(p, q, 0)$ if $A$ can be decomposed into the sum of two matrices $P$ and
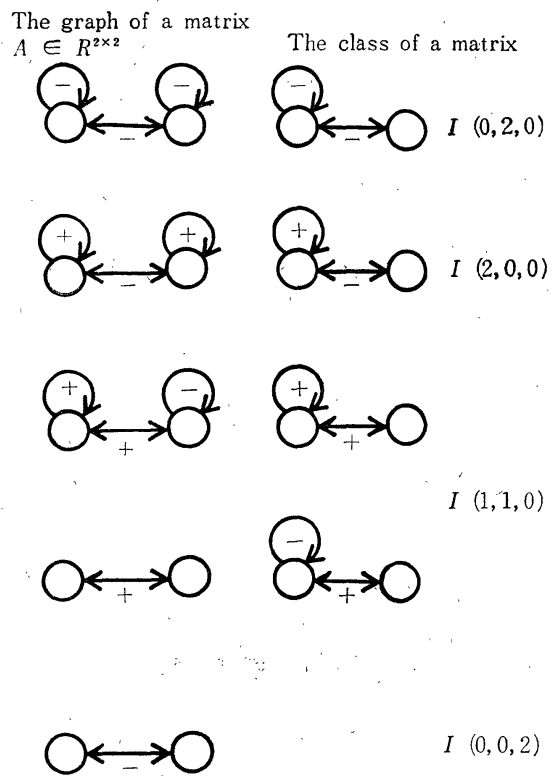

$I(0,0,2)$

Fig. 6-1 The graph of matrices $A \in R^{2 \times 2}$ which belong to $I(p, q, r)$

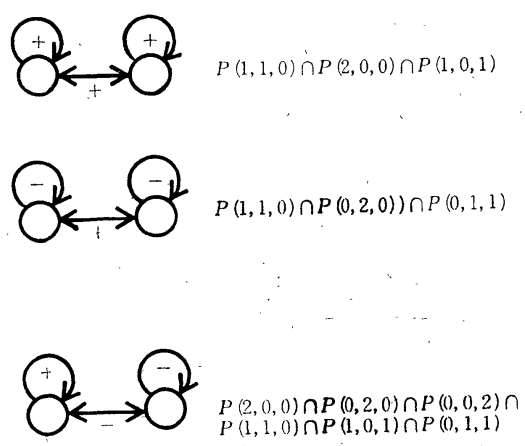

Fig. 6-2 The graph of matrices $A \in R^{2 \times 2}$ which belong to $P(p, q, r)$
$R, A=P+R$, where $P \in I(p, q, 0)$ is obtained by replacing some elements of $A$ by zero and $R=A-P$.

The above theorem can not be applied to matrix $A \in P(p, q, i),(i \neq 0)$ because $i$ will change even if the norm of the matrix $R$ is sufficiently small. Using these notation, sign stable matrices $A$ belong to $I(0, n, 0)$ and potentially stable matrices belong to $P(0, n, 0)$.

Fig. 6 shows how the matrices $A \in R^{2 \times 2}$ can be classified from the viewpoint of the sign pattern of $A$.

\section{Conclusion}

Throughout this paper, main theme is to see the qualitative feature of the solution of the system described by $d x / d t=A x$ only with its graphical structure. The method to check the sign stibility has been established and its analytical implication has been made clear with sign observability. The existing sufficient condition for potential stability is extended. However, necessary and sufficient condition for potential stability is not obtained. The concepts of sign stability and potential stability are generarized to that of inertia preservation of matrix. With the concept of inertia preservation the classification of qualitative matrices are discussed.

\section{References}

1) C. Jefferies: Qualitative Stability and Digraph in Model Ecosystem, Ecology 55, 1415/1419

2) C. Jefferies and V. Klee: When is a Matrix Sign Stable ?, Can. J. Math., 29-2, 315/326 (1977)

3) W.M. Anderson: Maximum Matching and the Rank of a Matrix, SIAM J. Appl. Math. 28, 114/123 (1975)

4) A. Ostrowski and H. Schneider: Some Theorems on the Inertia of General Matrices, J. Math. Anal. Appl. 4, 72/84 (1962)

5) J. Quirk: Qualitative Economics and the Stability of Equilibrium, Rev. Economic Studies, 32, 311/326 (1965)

6) J. Quirk: The Correspondence Principle, A Macroeconomic Application, Internat. Economic Rev., 9, 294/306 (1968)

7) H.K. Wimmer: On the Ostrowski-Schneider Inetia Theorem, J. Math. Anal. and Apll. 41, 164/169 (1973)

8) Y. Takeuchi : Stability Analysis of Systems of the Generalized Volterra Type, Ph. D. dissertation, Kyoto Univ. (1979) 


\section{《Appendix 》}

Appendix 1 (The proof of Theorem 1) (sufficiency)

Because of condition (2), $n$-positive constants $\lambda_{1}, \lambda_{2}, \cdots, \lambda_{n}$ can be defined so that $\lambda_{i} a_{i j}=-\lambda_{j} a_{j i}$ $(i \neq j)$. And due to condition (3), these constants can be defined uniquely if one of these constants is fixed with positive value. With these $n$-constants, we defined the positive quadratic form $V(x)$ :

$$
V(x)=\sum_{i=1}^{n} \lambda_{i} x_{i}^{2}
$$

Derivative of $V(x)$ along the solution of $d x_{i} / d t$ $=\sum_{j=1}^{n} a_{i j} x_{j}$ is $d V(x) / d t=2 \sum_{i=1}^{n} \lambda_{i} x_{i} d x_{i} / d t$ $=\sum_{i=1}^{n} 2 \lambda_{i} a_{i i} x_{i}^{2}$

Supposing that the matrix of the system has already been permuted into the form of condition (4), the time derivative of $V(x)$ can be described as follows :

$$
\begin{array}{r}
d V(x) / d t=2 \sum_{i=1}^{n} \lambda_{i} a_{i i} x_{i}{ }^{2} \quad a_{i i}<0 \text { for } i=1, \cdots, m \\
a_{i i}=0 \text { for } \\
i=m+1, \cdots, n
\end{array}
$$

Thus the solution of the system $d x / d t=\hat{A} x(\hat{A}$ $\left.\in Q_{A}\right)$ which satisfies $d V(x) / d t=0$ is of the form $\left(x_{1}, x_{2}, \cdots, x_{m}, x_{m+1}, \cdots, x_{n}\right)^{\imath}$ where $\left(x_{1}, \cdots, x_{m}\right)=(0$, $\cdots, 0)$. Therefore condition (4) implies that the solution which satisfies $d V(x) / d t=0$ is only the trivial solution $X=(0,0, \cdots, 0)^{t}$. So the time derivative $d V(x) / d t$ along every nonzero solution $X$ of $d x / d t=\hat{A} x$ is negative for every $\hat{A} \in Q_{A}$. And $V(x)$ can be considered as the Lyapunov function of the system.

(necessity)

In the characteristic equation of matrices $\hat{A} \in Q_{A}$, $\lambda^{n}+k_{1} \lambda^{n-1}+k_{2} \lambda^{n-2}+\cdots+k_{n-1} \lambda+k_{n}=0, k_{i}(i=1, \cdots, n)$ must be positive from the Routh-Hurwitz criterion. The condition (1) and (2) follows from the fact that $k_{1}=-\sum_{i=1}^{n} a_{i i}, k_{2}=\sum a_{i i} a_{j j}-\sum_{i \neq j} a_{i j} a_{j i}$ must be positive for every $\hat{A} \in Q_{A}$. And condition (3) is due to the determinantial condition of the Routh-Hurwitz criterion, det $H_{n}>0$ for every $\hat{A} \in Q_{A}$ where $H_{n}$ is the matrix of the form: $\left[\begin{array}{llll}k_{1} & k_{3} & \cdots & 0\end{array}\right.$

$$
\left[\begin{array}{cccc}
k_{1} & k_{3} & \cdots & 0 \\
1 & k_{2} & \cdots & \cdot \\
0 & & & \cdot \\
\vdots & & & \\
0 & & & k_{n}
\end{array}\right]
$$

for if there exists the cycle of the length greater than two then $\operatorname{det} H_{n}$ has the negative term (see 5) as for details). The condition (4) is also necessary because without it there exist the solution $x \neq 0$ such that $d V(x) / d t=0$ this the system fails to be stable.

Appendix 2 (The proof of the Theorem 4)

We use the next theorem to prove the Theorem 4. (As for the proof of this theorem, see 4))

《Theorem (Ostrowski-Schneider)》

If the matrix $H$ is a Hermitian solution of $A^{*} H$ $+H A=C$ where $C$ is the positive definite matrix then $\operatorname{In}(A)=\operatorname{In}(H)$.

(The proof of the Theorem 4)

If $E_{p q} A$ is sign stable then there exist some positive diagonal matrix $D$ such that $A^{t} E_{p q} D+$ $D E_{p q} A=-E$ (see 8)). Clearly $-E_{p q} D$ is a Hermitian solution of the equation in the above theorem, therefore $\operatorname{In}(A)=\operatorname{In}\left(-E_{p q} D\right)=(q, p, 0)$ for every $\hat{A} \in Q_{A}$.

Appendix 3 (The proof of the Theorem 6)

Because of the non existance of the length greater than two and $a_{i j} a_{j i} \leqq 0$ for every $i \neq j$, the positive definite quadratic form $V(x)$ can be constructed in the same way as in the proof of the Theorem 1 (see Appendix 1). And moreover, $d V(x) / d t$ $=2 \sum_{i=1}^{n} \lambda_{i} a_{i i} x_{i}{ }^{2}=0$, for $a_{i i}=0$ for every $i$. Thus the solution $x \neq 0$ of this system always satisfies $V(x)$ $=$ constant, and the trajectory of the solution is on the eliptical orbit in the solution space. 